\title{
Investigation of the gold nanoparticles effects on the prostate dose distribution in brachytherapy: gel dosimetry and Monte Carlo method
}

\author{
Hossein Khosravi, PhD', Bijan Hashemi, PhD², Faezeh Rahmani, PhD³, Ahmad Ebadi, PhD' \\ 'Department of Medical Physics, Salamat Institute, Chamran Hospital, ${ }^{2}$ Department of Medical Physics, Tarbiat Modares University, \\ ${ }^{3}$ Department of Physics, K. N. Toosi University of Technology, Tehran, Iran
}

\begin{abstract}
Purpose: In this work, gold nanoparticles (GNPs) were embedded in the MAGIC-f polymer gel irradiated with the ${ }^{192} \mathrm{Ir}$ brachytherapy sources.

Material and methods: At the first plexiglas phantom was made as the human pelvis. The GNPs were synthesized with $15 \mathrm{~nm}$ in diameter and $0.1 \mathrm{mM}(0.0197 \mathrm{mg} / \mathrm{ml})$ in concentration by using a chemical reduction method. Then, the MAGIC-f gel was synthesized. The fabricated gel was poured into the tubes located at the prostate (with and without the GNPs) locations of the phantom. The phantom was irradiated with ${ }^{192} \mathrm{Ir}$ brachytherapy sources for prostate cancer. After 24 hours, the irradiated gels was read by using Siemens 1.5 Tesla MRI scanner. Following the brachytherapy practices, the absolute doses at the reference points and isodose curves were extracted and compared by experimental measurements and Monte Carlo (MC) simulations.

Results: The mean absorbed doses in the presence of the GNPs in prostate were $14 \%$ higher than the corresponding values without the GNPs in the brachytherapy. The gamma index analysis (between gel and MC) using 7\%/7 mm was also applied to the data and a high pass rate achieved ( $91.7 \%$ and $86.4 \%$ for analysis with/without GNPs, respectively).

Conclusions: The real three-dimensional analysis shows the comparison of the dose-volume histograms measured for planning volumes and the expected one from the MC calculation. The results indicate that the polymer gel dosimetry method, which developed and used in this study, could be recommended as a reliable method for investigating the dose enhancement factor of GNPs in brachytherapy.
\end{abstract}

J Contemp Brachytherapy 2016; 8, 5: 422-428 DOI: 10.5114/jcb.2016.63453

Key words: GNPs, ${ }^{192} \mathrm{Ir}$, MAGIC-f, Monte Carlo, prostate cancer.

\section{Purpose}

The object of radiotherapy is to concentrate adequate radiation doses within tumor volumes with less effect on normal tissue. Since radio resistant tumor cells (e.g. hypoxic) are difficult to remove completely by radiotherapy, the radiation modalities use the limited doses to minimize the radiation effect on the normal surrounding tissue of the targeted region and exposed to the irradiated beam. In order to solve this problem, the injection of high-atomic number $(Z)$ material into tumors has been studied by researchers, and the development of radiosensitizers and radioprotectors has been explored to improve the effect of radiotherapy [1]. Nanotechnology is an emerging technique to improve the cellular targeting and radio-sensitization. Nanoparticles (NPs) are not widely used to treat cancers. Using structural modifications, NPs can be used as carrier for chemotherapeutic agents or radio-sensi- tizers to malignant cells [2]. Recently, the effect of gold nanoparticles (GNPs) in common radiotherapy has extensively been studied by using experimental measurements and Monte Carlo (MC) simulations. The idea of increasing the dose using some high- $Z$ elements has been proposed several decades ago, but following the invention and compatibility of GNPs with biological systems, scientists have been encouraged to investigate further applications of such elements in radiotherapy.

The increased absorbed doses in various tumors in the presence of GNPs have been confirmed by the most studies. However, the results are still controversial regarding the involved and prominent interaction processes of ionizing radiation with the GNPs, which have effects on dose enhancement factor (DEF) in tumor. Until now, the most effective parameters have been investigated such as the dimension of nanoparticles, high molar concentrations, and the lower energies of the photons or gamma rays to

\footnotetext{
Address for correspondence: Hossein Khosravi, PhD, Salamat Institute, Chamran Hospital, Tehran, Iran, Department of Medical Physics, Tarbiat Modares University, Al-Ahmad and Chamran, 14115-111 Tehran, 
obtain the higher doses by experimental dosimetry and MC methods $[3,4,5,6,7,8,9,10,11,12,13,14,15,16,17,18,19]$.

Monte Carlo methods are considered as the rigorous methods to estimate the possible macroscopic dose enhancement during the gold nanoparticles radiation therapy (GNRT) with the low-energy gamma-/X-ray sources such as ${ }^{125} \mathrm{I}, 50 \mathrm{kVp}$, and ${ }^{169} \mathrm{Yb}$ [11]. Monte Carlo methods are also applied to demonstrate significant changes in the spectra and fluency of the photo/Auger electrons within a GNPs loaded tumor during $x$-ray irradiation by the mentioned sources, which can be correlated well with the tumor dose enhancement [12].

Leung et al. investigated the characteristics of secondary electrons generated by the interaction of GNPs with X-rays regarded as nanoparticles size and beam energy leading to the GNP-enhanced radiotherapy. Their results could provide in the insights about the spatial distributions and the elevated dose in GNP-enhanced radiotherapy. They concluded that the irradiation of GNPs at the lower photon energies will be more efficient for cell killing [13].

Conventional dosimeters such as ion chambers, TLDs, and diode scan could measure only the point doses in the limited conditions. Various dosimetry methods are often used to confirm radiotherapy precisions. In brachytherapy, it is impossible to use such conventional dosimetry methods, especially in evaluation of the complex dose distributions resulting from interstitial practices. A large number of point dosimeters with very small sizes would be needed to put around the radiation sources, due to the high dose gradients. In addition, the dose values are highly dependent on the position and the small movements in dosimeters may lead to the significant dosimetric errors. Moreover, some of the conventional dosimeters are not tissue-equivalent, so they cause the distortions in the radiation field and dose distributions. Using the film dosimeters, radiation dose distributions could be measured only in a two-dimensional surface. Researchers have been motivated by these restrictions to use various gel polymers to measure complex dose distributions in radiotherapy $[20,21,22,23]$.

The MAGIC-f gel, as an appropriate three-dimensional and tissue-equivalent dosimeter, has been successfully used to determine the quantitative DEFs resulting from

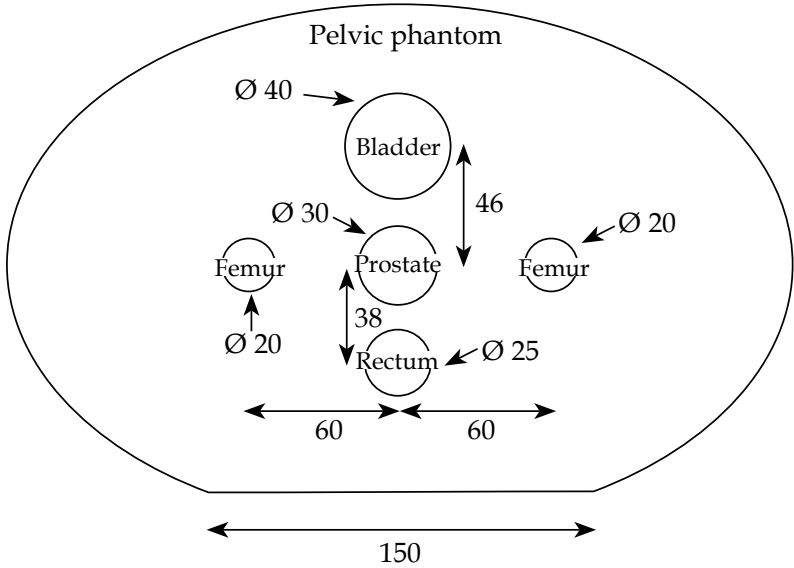

Fig. 1. The dimensions of pelvic phantom (the sizes of millimeters) the presence of GNPs as a compatible radio-sensitizers in various target organs. It could be potentially used to assess the effects of using GNPs in radiotherapy practices [24]. Recently, a polyacrylamide gel (nPAG) has been also used to measure the increase of the absorbed dose rate in the presence of GNPs at different energy ranges [25]. Overall, the gel dosimetry is reported as an appropriate tool for accurate dosimetric measurements when the nanoparticles are used in radiotherapy practices especially at $\mathrm{kV}$ energies.

Dosimetry evolved in brachynanotherapy is complex. So, the precise investigations of changes in dose due to nanoparticles could be determined using gel dosimetry. Brachynanotherapy conditions could be experimentally simulated incorporating GNPs to tissue-equivalent dosimeters. In this study, the effect of GNPs on the DEFs in the prostate under ${ }^{192}$ Ir brachytherapy sources has been experimentally investigated by using the MAGIC-f gel dosimeter and by MC simulations.

\section{Material and methods}

\section{Design and construction of the pelvic phantom}

The constructed plexiglas pelvic phantom has an elliptical shape with an overall dimension of $200 \times 200 \times 300 \mathrm{~mm}^{3}$, in which the positions of the human bladder, prostate, rectum, and bone tissue are defined (Figure 1). The bladder, prostate, and rectum incorporated in the phantom have a hollow cylindrical form to be poured with the desired gel. It should be noticed that two different caps were constructed for the prostate. The designed cap for the internal radiotherapy has appropriate holes to accommodate and to hold the brachytherapy needle sources. The phantom was designed and constructed according to the model made by CIRS company (Model 002PRA, Computerized Imaging Reference Systems, USA). Figure 2 shows implement of the treatment planning by after loader FLEXITRON system (Nucletron, Elekta company, Elekta AB, Stockholm, Sweden) in the Pars hospital. To obtain the gel dosimeter calibration curve, small plastic falcon tubes with a $20 \mathrm{~mm}$ in outer diameter, $100 \mathrm{~mm}$ in height, and $17 \mathrm{ml}$ in volume were used.

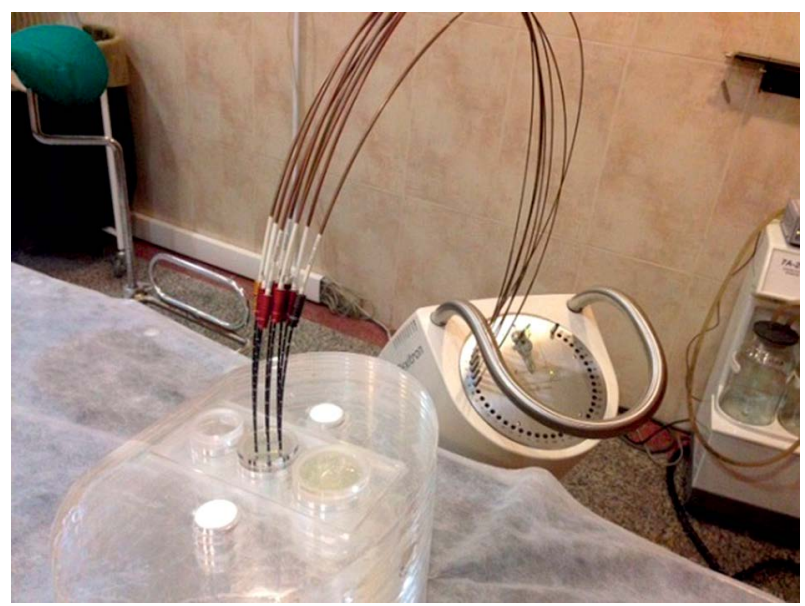

Fig. 2. Implement of treatment planning by the after loader FLEXITRON system in the Pars hospital 


\section{Gold nanoparticles synthesis}

The GNPs were produced using the gold salt with chemical reduction method as proposed by Kupiec et al. [26]. The gold nanoparticles were synthesized by sodium citrate reduction method with some modifications. The synthetic method developed in this experiment produces consistently steady gold nanoparticles, when the conditions are controlled properly. In this regard, a $100 \mathrm{~cm}^{3}$ aqueous solution containing $100 \mathrm{ppm} \mathrm{HAuCl}_{4}$ put in a round-bottomed flask under reflux condenser and heated up to $100^{\circ} \mathrm{C}$. Then, $1 \mathrm{~cm}^{3}$ trisodium citrate added at once and the mixture kept at this temperature for 2 hours without stirring. Just after the mixing, the color of the reaction mixture was light yellow, which changed into pellucid black after several minutes. Thereafter, the color of the mixture solution was slowly turned into ruby red color indicating the GNPs formation. Finally, the GNPs synthesized with appropriate amounts of its components to reach a diameter of $15 \pm 5 \mathrm{~nm}$. The average size of produced GNPs was proved by using the DLS (Dynamic Light Scattering, Malvern, UK) and TEM (Transmission Electron Microscopy) tests.

\section{Incorporating the gold nanoparticles in the gel}

The amount of $500 \mathrm{ml}$ of the MAGIC-f gel was fabricated (based on its original constituents [27]). In this study, a method based on several gel tubes was used to calibrate the gel dosimeter, in which several calibration tubes were put at $10 \mathrm{~cm}$ depth of a big container filled with distilled water to provide a uniform medium around them when irradiated. A separate tube was also kept outside as a controller when the other tubes were irradiated at known levels of 1, 2, 3.5, 6, and 9 Gy doses provided by the Varian 2100 Linac (Varian Medical Systems, Inc., Palo Alto, CA, USA). To assure the accuracy and reproducibility of the dosimeter calibration, the above process

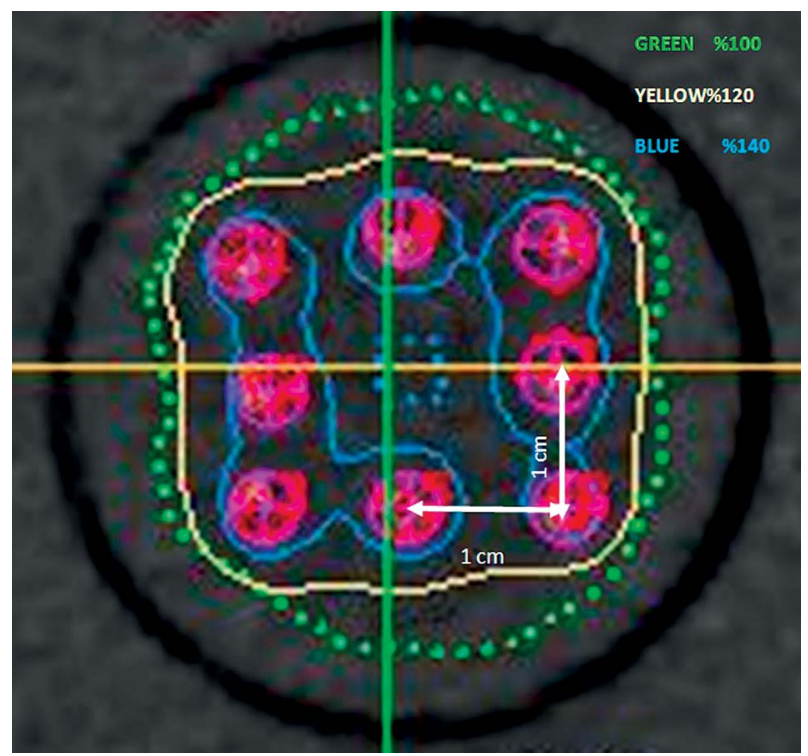

Fig. 3. The treatment planning performed on the pelvic phantom in the internal brachytherapy using ${ }^{192}$ Ir sources was repeated three times, and the relevant mean values were calculated and used against the dose levels.

The final MAGIC-f gel solution was divided into two separate volumes of 100 and $400 \mathrm{ml}$. Then, the volume portion of $100 \mathrm{ml}$ was transferred to another container and the GNPs solution was added to obtain a $0.1 \mathrm{mM}$ concentration of the GNPs in the final gel mixture. The volume of GNPs was calculated with use of relation $C_{1} V_{1}$ $=\mathrm{C}_{2} \mathrm{~V}_{2}$. That is $\mathrm{C}_{1}$, the first GNPs solution; $\mathrm{C}_{2}$, concentration of the GNPs in the final gel mixture; $\mathrm{V}_{1}$, volumes of the first GNPs solution; $V_{2}$, volume of the GNPs in the final gel mixture. The gel mixture was stirred for 5 minutes. Thereafter, the gel was poured in the calibration tubes, the prostate (with and without the presence of GNPs) and containers of the pelvic phantom, and it was kept in a refrigerator at $4^{\circ} \mathrm{C}$.

\section{Treatment planning and irradiation}

In brachytherapy, the required dose was selected from the planning target volume (PTV) defined by the radiotherapist. The Flexiplan software (Nucletron, Elekta company, Elekta AB, Stockholm, Sweden) was used for our brachytherapy treatment planning to compute the location of the source (dwell-position) and the amount of the time at each stop location (dwell-time). In the treatment planning for this radiotherapy procedure, the isodose curves were drawn for all images and computed tomography (CT) slices. A view of the isodose curves has been shown for this procedure in Figure 3. In the Paris system, the dose specification is based on an isodose surface called the reference isodose. However, in practice, the value of the reference isodose is fixed at $85 \%$ of the "basal dose", which is defined as the average of the minimum dose between sources [28].

Prior to implement the brachytherapy planning and to calibrate our fabricated MAGIC-f gel, the tubes containing the gel were irradiated in different defined doses fields. This calibration was performed by using calibrated dosimeter, which was provided by the Varian Linac. After calibrating the MAGIC-f gel, the phantom was irradiated with ${ }^{192}$ Ir sources as the high dose rate brachytherapy. The amount of delivered total dose was $4 \mathrm{~Gy}$.

\section{Magnetic resonance imaging reading and processing}

24 hours after the irradiation procedures, the gels were read by using a 1.5 Tesla Siemens MRI scanner (Siemens AG, Munich, Germany). Prior to the image processing, the phantom and calibration tubes were kept in an isothermal room for 4 hours.

Magnetic resonance images of both phantoms were acquired in the center of the head coil by using a multi spin-echo sequence with echo times of $22 \mathrm{~ms}$, repetition time of $3000 \mathrm{~ms}$, field of view of $300 \mathrm{~mm}$, and matrix size of $256 \times 256$ pixels (pixel size of $0.9 \times 0.9 \mathrm{~mm}^{2}$ ). For a straight comparison between measured and $\mathrm{MC}$ calculated data, the selected space between slices was $4 \mathrm{~mm}$, and the MRI images were acquired at the same positions as the brachytherapy slices.

The matrices plotted from the MRI R2 signals for each section were calculated by MATLAB software (version R2012b). The absolute doses and relative dose distribu- 
tion curves were extracted from the R2 signals of the MRI images.

\section{Monte Carlo simulation}

The MCNP5 code was used for the MC calculations. After validating the ${ }^{192}$ Ir sources (based on the TG-43 protocol [29]), the pelvic phantom was simulated according to its actual dimensions. In general, five materials including MAGIC-f gel, GNPs, Teflon (representing the femur bone) air (representing the rectum), and Plexiglas (representing the soft tissue of the rest of the pelvic phantom) were simulated in the pelvic phantom.

The simulated phantom was irradiated based on the same conditions as well as the protocols that explained in the experimental treatment planning procedures. The cutoff energy for the photons and electrons were set to $10 \mathrm{keV}$. F6 tally was used to calculate the doses deposited at different distances on the $\mathrm{X}$-axis. The voxel size of dose-scoring was $1 \times 1 \times 1 \mathrm{~mm}^{3}$. The number of photon histories for each simulation was set at $4 \times 10^{7}$ to ensure a statistical uncertainty less than $3 \%$ for all the dose scoring cells.

\section{Results}

The results are presented for experimental measurements as well as $\mathrm{MC}$ calculations according to the brachytherapy procedure described in the previous sections. At the experimental phase, the calibration process of the gel showed the linear relationship between the MRI $\mathrm{R} 2$ values and the doses, which were received by the calibration gel tubes (Figure 4).

The isodose curves have been presented in Figure 5 for prostate (with and without the presence of the GNPs) experimental measurements and the MC simulations in the pelvic phantom at the medial slice (located at $X=0 \mathrm{~cm}$ ) for the brachytherapy procedure, respectively.

The quantitative comparisons were performed by gamma index analysis using $7 \% / 7 \mathrm{~mm}$. The reason for choosing this criterion was an increase in acceptable pix-

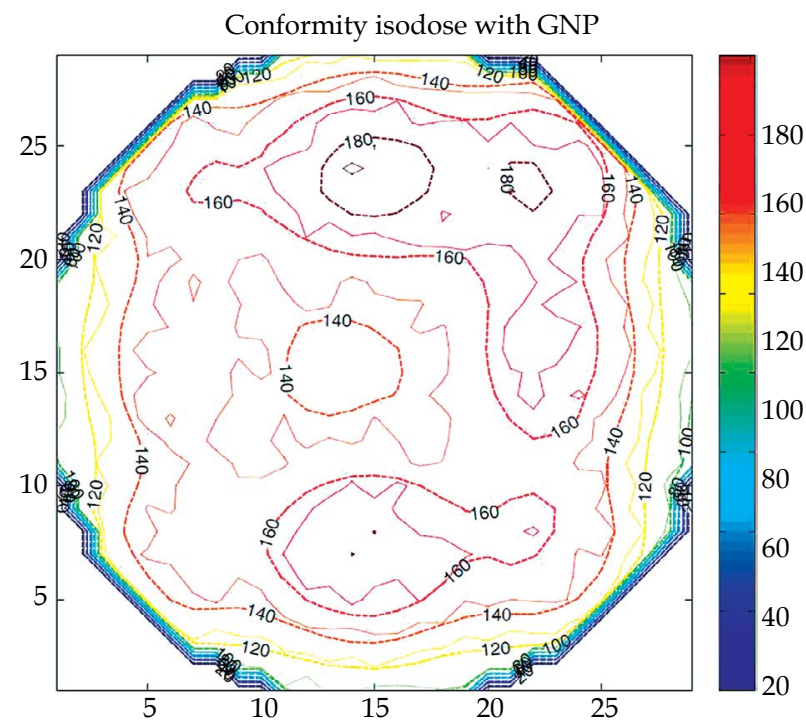

el frequency (with a gamma index smaller than one) by considering maximum acceptable error in brachytherapy. The results for the medial slice were presented as a histogram, which is showing the distribution of gamma values across the images (Figure 6). The gamma analyses, for the isocenter plane image with a high pass rate of $91.7 \%$ and $86.4 \%$, with/without GNPs, showed good agreement between the measurement and MC calculation results. It is also important to consider that the gamma analysis was performed across the entire image with doses values normalized to the isocenter dose value.

Dose-volume histograms (DVHs) were calculated to verify real three-dimensional dose distribution. A comparison between the measured data and calculated results (for the medial slice-with/without GNPs) in the gel is presented in Figure 7. For the PTV, the measured DVH showed that the regions doses are smaller than the expected doses from the MC simulation. The calculated mean relative dose values by MC were $147.9 \% \pm 0.1 \%$ (with GNPs) and $129.6 \% \pm 0.1 \%$ (without GNPs), while the measured mean relative dose value by gel was $141 \%$ $\pm 7 \%$ (with GNPs) and $124 \% \pm 5 \%$ (without GNPs).

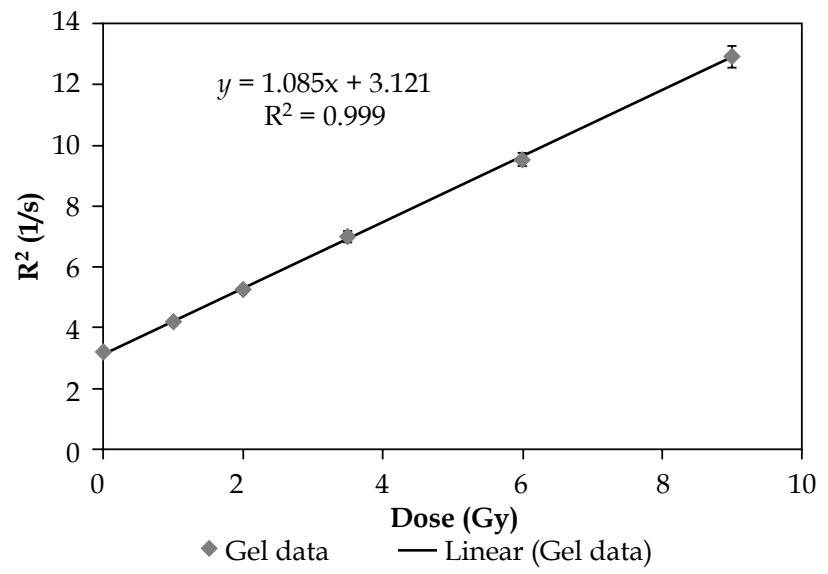

Fig. 4. The MAGIC-f gel calibration curve determined from the experimental measurements

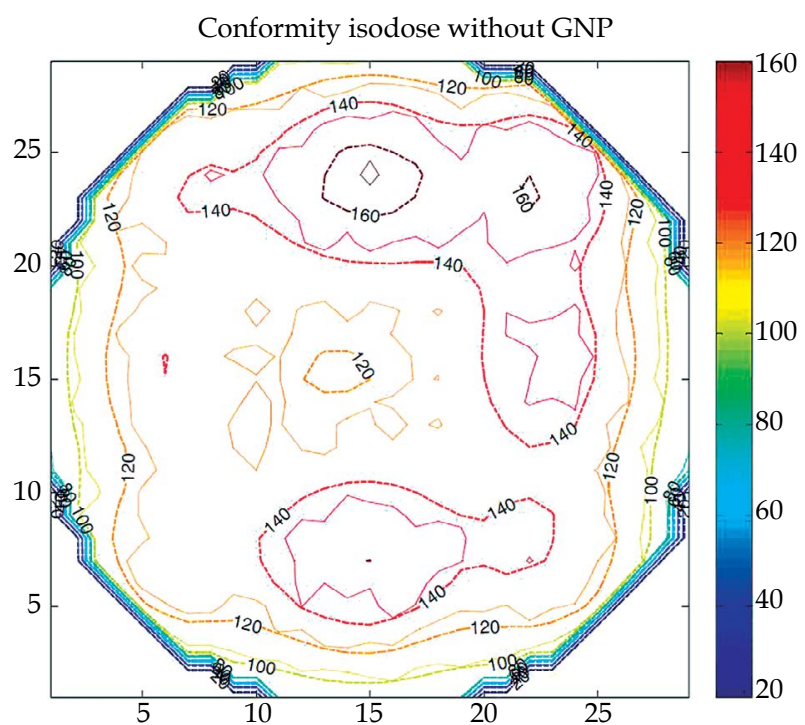

Fig. 5. Comparison of the isodose curves (e doses) measured experimentally with the gel (solid lines) and calculated by the MC calculations (dash lines) for brachytherapy 

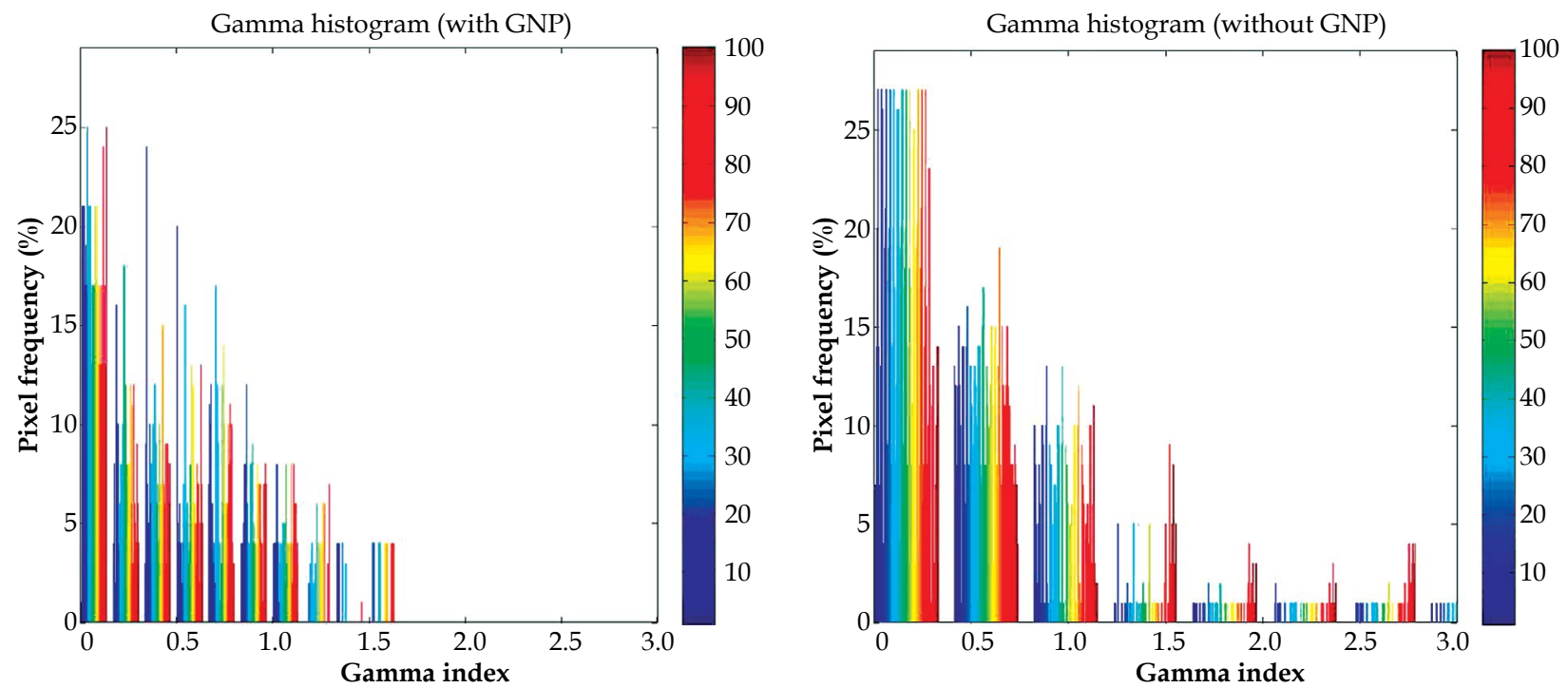

Fig. 6. Gamma images resulting of the 7\%/7 mm evaluation (with and without gold nanoparticles)
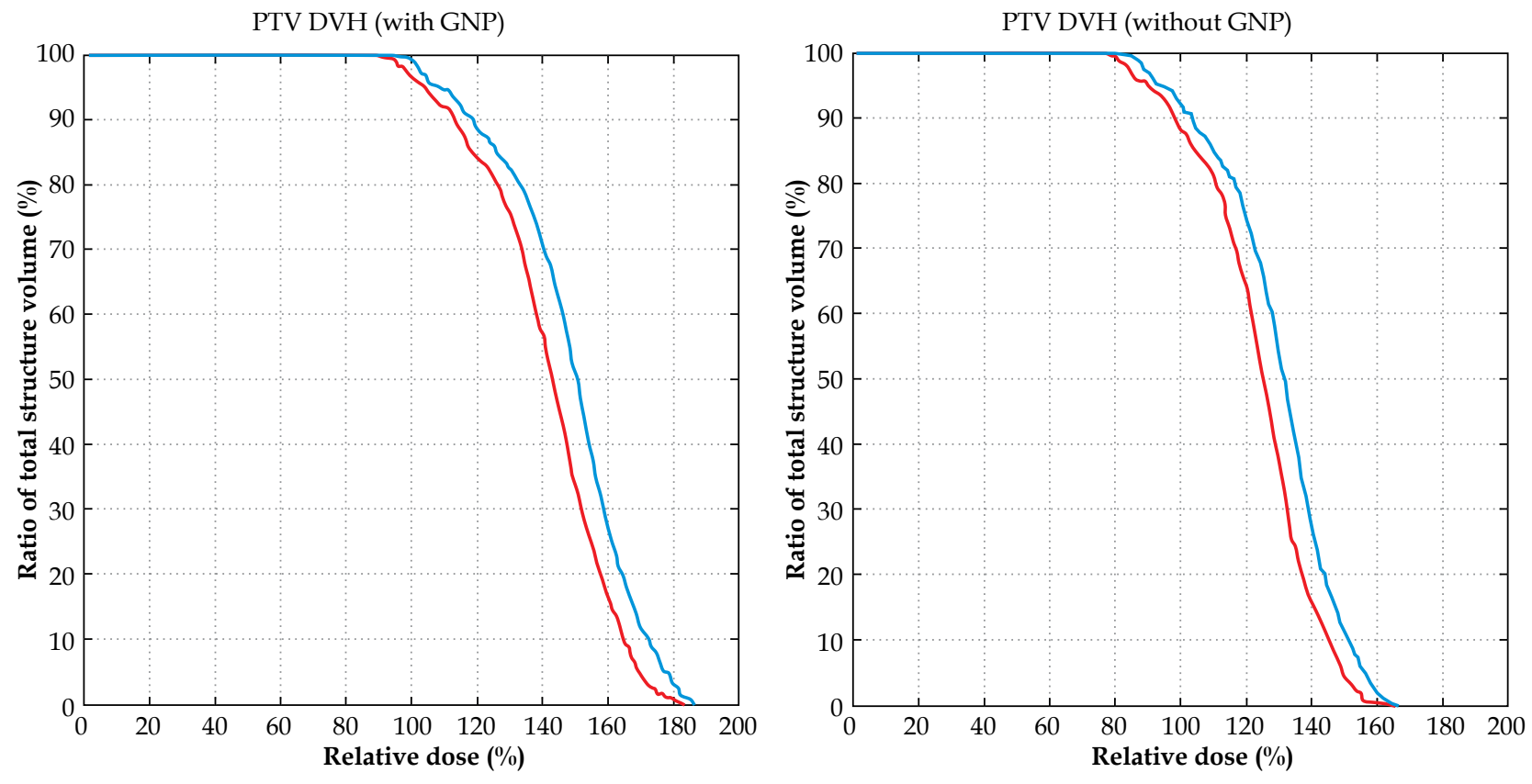

Fig. 7. Cumulative dose-volume histograms for the brachytherapy measured with gel and calculated by MCNP5 code for planning target volume

\section{Discussion and conclusions}

In the experimental part of this study, the linearity of the fabricated MAGIC-f gel dosimeter has been assessed. The fabricated gel showed a linear dose response in a range of 0-9 Gy with a considerable increase up to $50 \%$ that indicated on satisfactory and good polymerization process. The dose response of the gel has been also assessed with and without the presence of the GNPs for the brachytherapy, which is commonly used for the prostate cancer treatment in a pelvic phantom. Our experimental results in cases of the gel dosimeter indicated a mean
$\mathrm{DEF}=14 \%(\mathrm{SD}=3 \%)$ due to the presence of the GNPs in the prostate for the brachytherapy. In the brachytherapy, should be the higher probabilities of the photoelectric effect. Furthermore, the ratio of the mass absorption coefficient $\left(\mu_{\mathrm{en}} / \rho\right)$ of the gold to water at low energy is higher than that in the high energy $[19,30]$.

We have successfully simulated a MCNP5 Monte Carlo model by using nanoparticles geometry to study the dose enhancement induced by gold nanoparticles. The lattice property of MCNP5 code was employed in order to simulate the spherical gold nanoparticles inside the tumor uniformly. Three levels of lattice definitions were used. 
The first level was cells with a size of $1 \times 1 \times 1 \mathrm{~mm}^{3}$ inside a tumor. The second level was the cells (micrometer size) with dimensions of $0.001 \times 0.001 \times 0.0001 \mathrm{~mm}^{3}$, which uniformly fills the first level cells. And the third level was a spherical GNPs that fills each micrometer size cells inside the tumor. The first and second level cells were filled with gel. With this model, the effect of gold nanoparticles on radiation dose enhancement has been estimated more accurately. By modeling nanoparticles with realistic geometry, various radiation dose parameters, such as radiation energy and nanoparticles geometry as well as different concentrations have been studied to optimize the radiation dose enhancement effect. This method would provide valuable guidelines for future lab studies and clinical applications.

The measured gel dose distribution agreed with the expected results by the MC. The minor differences could be attributed to the image processing during registration of the images prior to the comparison, since the images had initially different resolutions, and the resolution of MC dose matrix is considerably lower in comparison to the measurement. Processing method to acquire the same resolution could add uncertainties to the altered image. Gel dosimeter measurement could provide a high-resolution dose distribution measurement, and could be better evaluated by comparison with a high resolution reference dose distribution, which in practice, is not available.

A complete three-dimensional dose distribution measurement of a brachytherapy prostate plan, which is using MAGIC-f gel dosimeter has been presented. The comparison of gel measurements with MC calculation showed a good agreement, especially in the region of the PTV. The results demonstrated that the fabricated MAGIC-f gel could be used as a suitable three-dimensional dosimeter for more extensive studies at various internal radiotherapy modalities in the presence of various concentrations of GNPs.

\section{Acknowledgments}

This study was extracted from a PhD project carried out by the first author, and with the help, advice and cooperation of other co-authors at Tarbiat Modares University, KNT University, and with the collaboration of Pars Hospital in Tehran, Iran. Hence, we would like to express our great appreciations to them for providing financial, technical, and clinical supports required for carrying out this research.

\section{Disclosure}

Authors report no conflict of interest.

\section{References}

1. Hainfeld JF, Dilmanian FA, Slatkin DN et al. Radiotherapy enhancement with gold nanoparticles. J Pharm Pharmacol 2008; 60: 977-985.

2. Kawasaki ES, Player A. Nanotechnology, nanomedicine, and the development of new, effective therapies for cancer. Nanomed Nanotechnol 2005; 1: 101-109.

3. Hainfeld JF, Slatkin DN, Smilowitz HM. The use of gold nanoparticles to enhance radiotherapy in mice. Phys Med Biol 2004; 49: 309-315.
4. Rahman WR, Bishara N, Ackerly T et al. Enhancement of radiation effects by gold nanoparticles for superficial radiation therapy. Nanomed Nanotechnol 2009; 5: 136-142.

5. Asadi S, Vaez-zadeh M, Masoudi F et al. Gold nanoparticle-based brachytherapy enhancement in choroidal melanoma using a full Monte Carlo model of the human eye. J Appl Clin Med Phys 2015; 16: 344-357.

6. Spirou SV, Makris D, Loudos G. Does the setup of Monte Carlo simulations influence the calculated properties and effect of gold nanoparticles in radiation therapy? Phys Med 2015; 31: 817-821.

7. Wolfe T, Chatterjee D, Lee J et al. Targeted gold nanoparticles enhance sensitization of prostate tumors to megavoltage radiation therapy in vivo. Nanomedicine 2015; 11: 1277-1283.

8. Kuban DA, Tucker SL, Dong L et al. Long-term results of the M. D. Anderson randomized dose-escalation trial for prostate cancer. Int I Radiat Oncol Biol Phys 2008; 70: 67-74.

9. Zhang SX, Gao J, Buchholz TA et al. Quantifying tumor-selective radiation dose enhancements using gold nanoparticles: A Monte Carlo simulation study. Biomed Microdevices 2009; 11: 925-933.

10. Zhang XD, Guo ML, Wu HY et al. Irradiation stability and cytotoxicity of gold nanoparticles for radiotherapy. Int J Nanomedicine 2009; 4: 165-173.

11. Cho SH, Jones BL, Krishnan S. The dosimetric feasibility of gold nanoparticle-aided radiation therapy (GNRT) via brachytherapy using low-energy gamma-/X-ray sources. Phys Med Biol 2009; 54: 4889-4905.

12. Cho SH. Estimation of tumor dose enhancement due to gold nanoparticles during typical radiation treatments: a preliminary Monte Carlo study. Phys Med Biol 2005; 50: 163-173.

13. Leung MKK, Chow JCL, Chithrani BD et al. Irradiation of gold nanoparticles by x-rays: Monte Carlo preliminary simulation of dose enhancements and the spatial properties of the secondary electrons production. Med Phys 2011; 38: 624-631.

14. Ngwa W, Korideck H, Kassis AI et al. In vitro radiosensitization by gold nanoparticles during continuous low-dose-rate gamma irradiation with I-125 brachytherapy seeds. Nanomedicine 2013; 9: 25-27.

15. Su XY, Liu PD, Wu H et al. Enhancement of radiosensitization by metal-based nanoparticles in cancer radiation therapy. Cancer Biol Med 2014; 11: 86-91.

16. McMahon SJ, Mendenhall MH, Jain S et al. Radiotherapy in the presence of contrast agents: a general figure of merit and its application to gold nanoparticles. Phys Med Biol 2008; 53: 5635-5651.

17. Khosravi H, Hashemi B, Mahdavi SR et al. Effect of Gold Nanoparticles on Prostate Dose Distribution under Ir-192 Internal and 18 MV External Radiotherapy Procedures Using Gel Dosimetry and Monte Carlo Method. J Biomed Phys Eng 2015; 5: 3-14.

18. Chow JCL, Leung MKK, Jaffray DA. Monte Carlo simulation on a gold nanoparticle irradiated by electron beams. Phys Med Biol 2012; 57: 3323-3331.

19. Roeske JC, Nunez L, Hoggarth M et al. Characterization of the theoretical radiation dose enhancement from nanoparticles. Technol Cancer Res Treat 2007; 6: 395-401.

20. IbbottGS. Clinical applications of gel dosimeters. In:LepageM, Jirasek A, Schreiner LJ (eds.). DOSGEL 2006 - Fourth international conference on radiotherapy gel dosimetry. University of Sherbrook, Sherbrook 2006; 237-267.

21. McJury M, Oldham M, Cosgrove V et al. Radiation dosimetry using polymer gels: methods and applications. Br J Radiol 2000; 73: 919-929.

22. Watanabe Y, Gopishankar N. Three-dimensional dosimetry of Tomo Therapy by MRI-based polymer gel technique. J Appl Clin Med Phys 2011; 12: 3273. 
23. Gopishankar N, Vivekanandhan S, Rath GK et al. Indigenously developed multipurpose acrylic head phantom for verification of IMRT using film and gel dosimetry. J Appl Clin Med 2013; 14: 62-76.

24. Marques T, Schwarcke M, Garrido C et al. Gel dosimetry analysis of gold nanoparticle application in kilovoltage radiation therapy. The $6^{\text {th }}$ International Conference on 3D Radiation Dosimetry, 2010 August 22-26; South Carolina, USA.

25. Rahman WN, Wong CJ, Ackerly T et al. Polymer gels impregnated with gold nanoparticles implemented for measurements of radiation dose enhancement in synchrotron and conventional radiotherapy type beams. Australas Phys Eng Sci Med 2012; 35: 301-309.

26. Kupiec AS, Malina D, Zimowska M et al. Characterization of gold nanoparticles for various medical applications. Digest Journal Nanomaterial \& Biostructures 2011; 6: 803-808.

27. Fernandes JP, Pastorello BF, de Araujo DB et al. Formaldehyde increases MAGIC gel dosimeter melting point and sensitivity. Phys Med Biol 2008; 53: N53-58.

28. Khan FM. The physics of radiation therapy. $4^{\text {th }}$ ed. Lippincott Williams and Wilkins, Philadelphia 2010; Chapter 15.

29. Rivard MJ, Coursey BM, DeWerd LA et al. Update of AAPM Task Group No. 43 Report: A revised AAPM protocol for brachytherapy dose calculations. Med Phys 2004; 31: 633-674.

30. Khoshgard K, Hashemi B, Arbabi A et al. Radiosensitization effect of folate-conjugated gold nanoparticles on HeLa cancer cells under orthovoltage superficial radiotherapy techniques. Phys Med Biol 2014; 59: 2249-2263. 